\title{
Anti-inflammatory effect of breads in relation to the bioavailability of phenolic compounds
}

\author{
N. Mateo Anson ${ }^{1,2}$, E. Selinheimo ${ }^{3}$, A.-M. Aura ${ }^{3}$, R. Havenaar ${ }^{1}$, A. Bast ${ }^{2}$ and G. R. M. M. Haenen ${ }^{2}$ \\ ${ }^{1}$ TNO Quality of Life, P.O. Box 360, 3700 AJ Zeist, The Netherlands, ${ }^{2}$ Maastricht University, P.O. Box 616, 6200 MD, \\ Maastricht, The Netherlands and ${ }^{3}$ VTT, P.O. Box 1000, Fi-02044 VTT, Finland
}

Phenolic compounds (PC) are food-derived bioactive compounds contained in the daily diet. PC have been claimed to help prevent cancer, degenerative diseases and chronic and acute inflammation. A possible mechanism of anti-inflammatory action is by their effect on the redox sensitive transcription factor nuclear factor kappaB, inducible nitric oxide synthase expression and cyclooxygenase inhibition. The anti-inflammatory effects of PC are evident in vitro using high concentrations; however, physiological concentrations are dependent on their bioavailability from the food source, which is often limited. Cereal products contribute substantially to the total intake of PC. Most of the PC in the cereal grain are concentrated in the outermost part, the bran. Therefore, wholemeal breads containing either native or bioprocessed bran were tested for their post-prandial effects on an inflammatory response ex vivo. The objective of this investigation was to evaluate the anti-inflammatory effect of PC in relation to their bioavailability from a natural dietary source, such as bread. The study design was: crossover, with 8 healthy men undergoing 3-days low phenolic diet prior to intervention and at least 1 week of wash-out period between the two intervention days. Blood was drawn before bread ingestion ( $t_{0}$ or baseline), and at $1 \mathrm{~h} 15 \mathrm{~min}, 6 \mathrm{~h}$ and $12 \mathrm{~h}$ after ingestion. Whole blood was incubated with LPS $(1 \mathrm{ng} / \mathrm{ml})$ for $24 \mathrm{~h}$ and TNF- $\alpha$, IL-6, IL-1 $\beta$ and IL-10 were determined. The PC quantified in plasma were: ferulic acid, vanillic acid, $p$-coumaric acid, sinapic acid and their colonic metabolites: phenylpropionic acids, phenylacetic acids, and benzoic acids with different grades of hydroxylation. The ratio of pro-/anti-inflammatory cytokines at $1 \mathrm{~h} 15 \mathrm{~min}, 6 \mathrm{~h}$ and $12 \mathrm{~h}$ after bread ingestion was lower than baseline $\left(t_{0}\right)$. The bread with bioprocessed bran showed a stronger effect than the one with native bran, which could be associated with an increase in the bioavailability of PC by the bioprocessing.
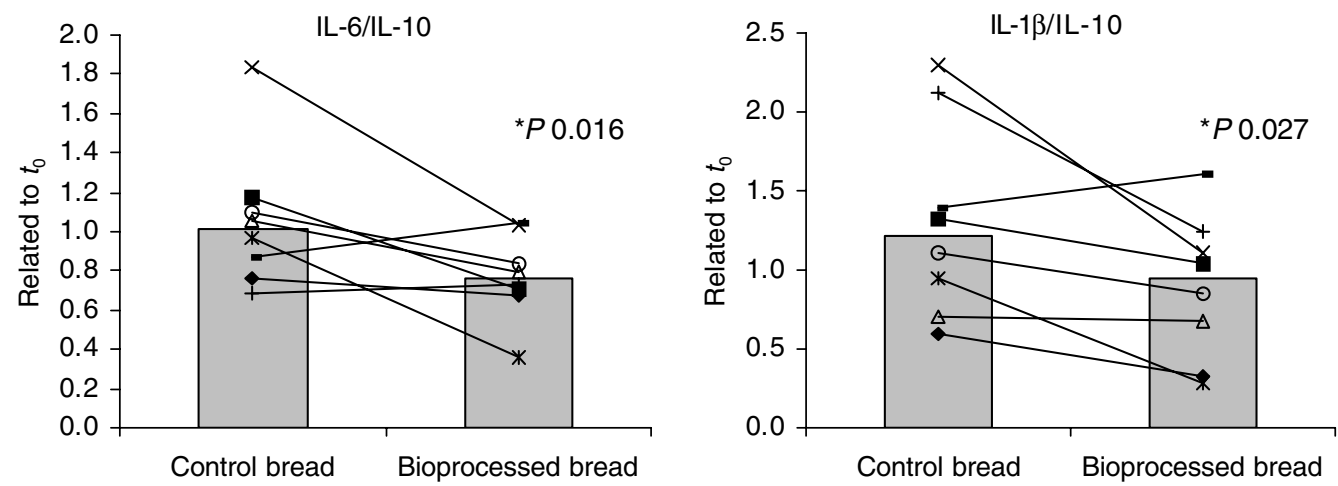

Fig. 1. Ratio of the pro-/anti-inflammatory cytokines: IL-6/IL-10 and IL-1 $\beta / \mathrm{IL}-10$ in LPS-stimulated blood collected at $1 \mathrm{~h} 15 \mathrm{~min}$ after ingestion of the bread. Each cytokine is related to its baseline value (LPS-stimulated blood collected at $\left.t_{0}\right)$. Bars show the median value $(n=8)$.

This work was financially supported by the European Commission in the Communities 6th Framework Programme, Project HEALTHGRAIN (FOOD-CT2005-514008). It reflects the author's views and the Community is not liable for any use that may be made of the information contained in this publication. 